\title{
The Impact of Computing Device Design on Patient-Centered Communication: An Experimental Study
}

\author{
Rebecca Randell* \\ School of Healthcare \\ University of Leeds \\ R.Randell@leeds.ac.uk
}

\author{
Charlotte Tang* \\ Department of Computer Science \\ University of Michigan-Flint \\ tcharlot@umflint.edu
}

\author{
Yunan Chen \\ Department of Informatics \\ University of California, Irvine \\ yunanc@ics.uci.edu
}

*Equal contributions

\begin{abstract}
Prior research has studied the impact of use of a single computing device, such as a desktop or a tablet computer, on patient-provider communication. While some studies have considered how contextual features such as room layout and software interface design affect computer use and patient-provider interaction in the exam room, it is not known how the choice of computing device impacts patient-provider communication. We conducted a within-participant experimental study. Three physicians participated in nine simulated consultations, using a desktop computer, a tablet computer, and a tabletop computer. Consultations were video-recorded and the video data were analyzed using framework analysis. Findings reveal the choice of device impacts the extent to which the consultation is patient-centered. To better support patient-centered communication, a large adjustable horizontal screen can facilitate eye contact and patient engagement. Findings also highlight the need for design of future systems to consider the characteristics of both openness and privacy.
\end{abstract}

\section{Introduction}

The Institute of Medicine defines patient-centered care as "care that is respectful of and responsive to individual patient preferences, needs, and values." [17]. This suggests effective communication is key to patient-centered care [11]. In fact, patient-physician communication has been widely considered to be critical to the success of patient care and is not limited to verbal discourse, but also nonverbal communication such as the tone of voice, posture, gesture, and facial expressions [15][18][24]. Both verbal and nonverbal communication can impact the interpersonal relationship between a physician and a patient.
Given the potential of health information technology (health IT) such as Electronic Medical Records (EMR) in improving the quality, efficiency, safety, and reducing the cost of health care, computing devices have been gradually introduced into healthcare settings. The ubiquitous use of computing devices in the exam room has thus resulted in a wealth of literature: some argued that computer use during consultations has the potential to enhance patient-centered care [42], while the majority of studies have highlighted the negative impacts of computer use on patient-centeredness [36]. A topic that has received particular attention is how computer use affects healthcare providers' gaze and bodily orientation, as turning to gaze at the patient demonstrates engagement and encourages patient participation in the consultation [3][4][12][26][29].

While previous research has considered how contextual features such as room layout and software interface design affect exam room computer use and subsequent impacts on patient-provider interaction [18][33], most of these studies have only examined the use of a single computing device, typically the desktop computer (e.g., [15]), during patient consultation. Less is known about how different devices impact patient-provider communication. One simulated study has examined the impact of a paper chart, a PDA, and a laptop mounted on a trolley on patient-provider communication in hospital ward rounds [1]. They identified a number of benefits afforded by the paper chart in comparison to the digital devices due to the user interface and form factor of the latter, and the personal characteristics of the physicians. For example, a paper chart allowed physicians and patients to more easily re-establish eye contact, interact verbally and non-verbally with gesture, retrieve information, and maintain mutual awareness of actions. To our knowledge, the present study is the first to explore the impact of different devices on patient-provider communication in the exam room. 


\section{Related work}

Patient-physician communication is a vital element in the care process. Thus, a large body of research has been conducted to investigate the behaviors during consultations. They found that the effectiveness of patient-provider communication could impact patient satisfaction, patient engagement, and health outcomes such as participatory decisionmaking and the reduction in medical errors [14][37]. In particular, prior research suggested that poor quality of communication during consultations was a major reason for tensions in the relationship between physicians and patients [22] and reduced trust between them [21]. A recent study also identified four barriers to improving patient-physician communication in the exam room: low medical literacy of patients, high workload for physicians, low awareness of communication skills, and adoption of defensive behaviors by physicians [40].

Health IT can potentially enhance the quality and efficiency of care through improved documentation and tracking of patient care activities [7]. Thus, computers and EMRs are implemented in healthcare settings to provide more efficient care and to reduce medical errors [6]. However, deploying health IT in clinical settings is challenging because of a variety of adoption barriers. For example, existing health IT systems were found to fall short in supporting frontline clinical processes [8][41]. Therefore, previous research recommended that health IT should be ergonomically suitable for the particular setting and seamlessly integrated into existing patient care practices to fit the ecology of situated practices [9][10]. In addition, it is important to be aware of unintended consequences that may occur with health IT use during the process of entering and retrieving information and during the communication and coordination process that the technology is meant to support [5].

The introduction of computing devices into the exam room impacts the interaction between patients and physicians. Specifically, computer use during patient-physician interaction created a barrier to communication when the screen is only visible to the physician, and could thus impact their verbal exchange, gazes, and relative orientations [12]. However, the computing device could serve as a shared artifact for information exchange if the screen was re-oriented so that both physician and patient could see [12]. Patients were also found to feel anxious when physicians were recording information on the computer instead of gazing at the patients during consultations. On the other hand, physicians perceived that allowing patients to view the screen when recording information at the computer could lead to unnecessary anxiety. Therefore, physicians must be careful with the amount and the type of information to be displayed, particularly when sharing sensitive data, so as not to increase patients' anxiety [28].

Most prior studies have examined the use of desktop computers in the exam room and their impact on patient-physician communication (e.g., [15][8]). As mobile devices are making their way into the exam room, research has been conducted to investigate patient's attitude towards their physicians' use of mobile devices, such as PDAs (e.g., [32]) and Tablet PCs (e.g., [39]), on patient-physician communication during consultations. Patients were generally positive towards the use of technology by their physicians, but many were concerned about privacy [20]. In addition, most of these previous studies focused on a single type of computing devices; only one studied the use of different devices in a hospital ward round [1]. The current research however conducted an experimental study to examine three different computing devices to investigate their impacts on patient-physician communication in the exam room.

\section{Methodology}

We conducted an experimental study to compare different computing devices used during simulated consultations in a laboratory environment. Simulated consultations were chosen due to the challenges of comparing these devices in situ, and simulated consultations have been effective in previous research to examine the impact of computer use on patient-provider communication [1][13][24].

\subsection{Data collection}

\subsubsection{Devices}

Three digital devices were used in the study:

- a traditional desktop computer which was not designed for easy rotating,

Table 1. Simulated Consultations Study Design

\begin{tabular}{l|l|l|l}
\hline & Physician 1 & Physician 2 & Physician 3 \\
\hline Scenario 1 & Desktop & Tabletop & Tablet \\
\hline Scenario 2 & Tablet & Desktop & Tabletop \\
\hline Scenario 3 & Tabletop & Tablet & Desktop \\
\hline
\end{tabular}


- a Panasonic Toughbook CF-H1 Mobile Clinical Assistant (MCA) tablet computer with a 10.4 inches screen using a digitizer pen for input, and

- a tabletop computer that requires mouse and keyboard input.

These three devices were selected for our study because the desktop computer was the most popular computing device used in exam rooms, the portable nature of tablet PC made it particularly suitable for physicians' mobility and dynamic work at the point of care, and the tabletop computer has recently made its way into healthcare settings.

\subsubsection{Simulation scenarios}

Three scenarios were developed with the help of a practicing primary care physician for the simulated consultations. In scenario 1 , the patient is seeking vaccinations information for working in Uganda. In scenario 2, the patient wants information about the measles, mumps, and rubella (MMR) vaccinations that his daughter is due to have. In scenario 3, the patient has a family history of cancer and wants information regarding diet and screening.

All of the scenarios involved information gathering by the physician and information sharing with the patient.

\subsubsection{Study design}

An experimental study was conducted with three primary care physicians participating in simulated consultations with patient actors presenting with symptoms based on the three scenarios described above. The physicians were aware that the patients were actors and they were asked to behave as if they were in consultation with real patients. The simulated consultations were video-recorded. Semi-structured interviews were then conducted with each physician after the study to solicit their opinions and experiences of the devices.

A within-participant design was used, with each participant using each of the devices by participating in three consultations with different scenarios as shown in Table 1.

\subsection{Data analysis}

Analysis followed the steps of framework analysis, a theme-based approach developed in the 1980s for analyzing qualitative data for the purpose of applied research [31]. Framework analysis was used to reduce the data through summarization and synthesis while retaining links to original data. The analysis outputs a matrix display.

Two authors first reviewed all the simulation videos to familiarize themselves with the data. From this, a thematic framework was developed for indexing the data, including phases of the consultation, physician gaze and bodily orientation, and ways in which the physician encouraged or discouraged the patient from screen sharing. The two authors then indexed instances of these behaviors in the videos, using the software package Transana (www.transana.org).

A matrix display [25] based on a typology from previous research: active information sharing (physician actively encourages patient to view the screen), passive information sharing (physician does not encourage patient to view the screen but does not prevent them from doing so), and technology withdrawal (physician keeps screen out of the patient's line of sight) [2][4] was created. The matrix was used to support within-participant comparisons (similarities and differences in the same participant's usage behaviors for devices) and between-participant comparisons (common usage behaviors for the same device across physicians).

Finally, another author independently reviewed all the videos and verified the indexed behaviors in the matrix.

\section{Findings}

Regardless of physician, device, or reason for the patient's visit, all consultations had the same basic structure: 1) before patient arrival, physician looked at patient's electronic health record; 2) physician asked patient about the reason for their visit; 3) physician provided information to patient, using the computer to search for relevant information; 4) physician concluded consultation by confirming next steps for either physician or patient; 5) when patient left the room, physician recorded details of consultation in the computer system.

In the remainder of this section, we focus on step 3 , since the remaining steps did not involve simultaneous patient-provider interaction and computer use. The matrix display in Table 2 summarizes the physicians' gaze, bodily orientation, and the extent to which they shared the screen with the patient varied according to the device used.

\subsection{Desktop computer}

Desktop computers are the most commonly used computing devices in the exam room. In our study, the computer was placed on a regular desk, with the 
Table 2. Physician's gaze, relative bodily and device orientation, and information sharing behaviors

\begin{tabular}{|c|c|c|}
\hline Desktop computer & Tablet computer & Tabletop computer \\
\hline \multicolumn{3}{|l|}{ Physician 1} \\
\hline 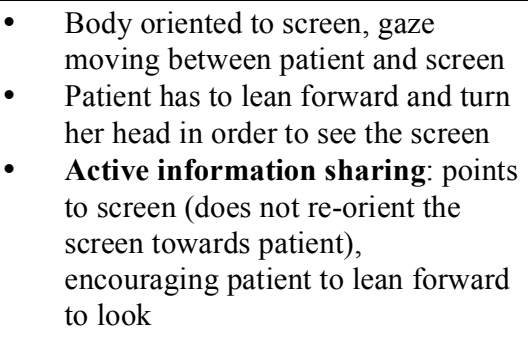 & $\begin{array}{l}\text { Body oriented to screen, gaze } \\
\text { moving between patient and screen } \\
\text { Active information sharing: } \\
\text { moves screen towards patient and } \\
\text { gestures towards screen }\end{array}$ & $\begin{array}{l}\text { - Gaze moving between patient and } \\
\text { screen but with more time looking } \\
\text { at screen in comparison to other } \\
\text { scenarios } \\
\text { Patient has side-view of screen, } \\
\text { gaze follows physician's gaze and } \\
\text { pointing } \\
\text { Active information sharing: } \\
\text { gesturing towards screen }\end{array}$ \\
\hline \multicolumn{3}{|l|}{ Physician 2} \\
\hline $\begin{array}{l}\text { Patient re-orients the chair toward } \\
\text { and beside doctor upon arrival so } \\
\text { patient can see the screen } \\
\text { Initially body oriented to screen and } \\
\text { gaze focused on screen, then sits } \\
\text { back to talk to patient, gaze focused } \\
\text { on patient and body oriented to } \\
\text { patient } \\
\text { Active (two-way) information } \\
\text { sharing: patient leans in and points } \\
\text { to screen, then physician says 'here } \\
\text { you are', further encouraging } \\
\text { patient engagement }\end{array}$ & $\begin{array}{l}\text { Body oriented to screen, gaze } \\
\text { moving between patient and screen } \\
\text { Technology withdrawal: } \\
\text { physician keeps screen close, } \\
\text { angles towards himself for sensitive } \\
\text { topic. }\end{array}$ & $\begin{array}{l}\text { Physician does not search for } \\
\text { information but looks at patient } \\
\text { record for details of previous } \\
\text { vaccinations, lower body oriented } \\
\text { to patient, leans forward to screen } \\
\text { Passive information sharing: } \\
\text { patient gaze follows physician } \\
\text { gaze to screen }\end{array}$ \\
\hline \multicolumn{3}{|l|}{ Physician 3} \\
\hline $\begin{array}{l}\text { Lower body oriented to patient, } \\
\text { gaze moving between patient and } \\
\text { screen } \\
\text { Active information sharing: } \\
\text { physically turns the monitor } \\
\text { towards the patient and points } \\
\text { towards information on screen }\end{array}$ & $\begin{array}{l}\text { Lower body oriented to patient, } \\
\text { gaze focused on screen as reading } \\
\text { out information, glances up when } \\
\text { responding to patient question } \\
\text { Technology withdrawal: } \\
\text { physician keeps screen close, } \\
\text { angles towards himself and away } \\
\text { from patient (for physician's own } \\
\text { use) }\end{array}$ & $\begin{array}{l}\text { Lower body oriented to patient, } \\
\text { gaze focused on screen but turns } \\
\text { to patient when responding to } \\
\text { patient question } \\
\text { Passive information sharing: } \\
\text { using mouse to highlight } \\
\text { information, patient also points to } \\
\text { information on screen }\end{array}$ \\
\hline
\end{tabular}

physician sitting in front of the desk, which is typical of most exam room set ups. The patient either sat next to the desk slightly facing the physician (Figure 1 , left \& center) or re-oriented the chair to be sitting orthogonal to the physician and be able to see the screen (Figure 1, right). Regardless, the relative positioning of the physician, the computer, and the patient made it difficult for the physician to give attention to both the patient and the screen, consistent with previous findings [1]. In all three consultations,
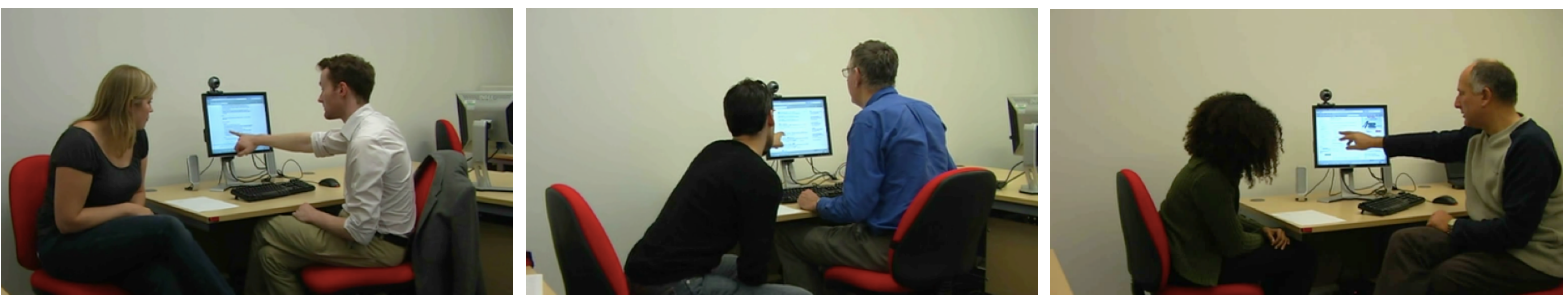

Figure 1: Different ways of active information sharing (left) Patient leans forward to follow physicians' pointing to see the screen, (center) Patient re-oriented the chair to view the screen and points to share information with physician, (right) Physician turned the screen towards patient and points at the screen. 
the physician had to frequently move his gaze between the screen and the patient regardless of where the patient sat, as shown in Figure 1. In addition, the monitor used in our study was not easy to rotate, so in only one consultation did the physician slightly angle the screen towards the patient. In the other consultations, the patient had to lean forward or re-orient the chair to see what was on the screen (Figure 1).

Active information sharing was demonstrated in all three consultations using a desktop computer: 1) physician did not turn the screen towards patient, but pointed at or gestured towards the screen to subtly encourage the patient to lean forward to look at the screen, 2) patient re-oriented the chair so as to see the screen and to point at the screen to share information with the physician who also made verbal comments like "here you are" to guide patient to see the screen, and 3) physician turning the screen toward patient and pointing at the screen to share information with patient actively.

Therefore, desktop computers make it difficult for the physician to make eye contact with the patient. With its relatively large, vertical screen, information sharing can take place actively by the physician turning the screen towards the patient and pointing or gesturing to direct patient's gaze at the screen. Alternatively, patients who are keen on seeking information can actively point and gesture on the screen.

\subsection{Tablet computer}

Tablets are known for their small and portable nature and we anticipated that this would make it easy for the physician to share the screen with the patient. The small size of the tablet did afford more control and flexibility, with all three physicians moving the device during the consultations. However, both physicians 2 and 3 only moved the screen towards themselves, rather than re-orienting it to show the screen to the patient, demonstrating a behavior we refer to as technology withdrawal (Figure 2).
The reason for technology withdrawal could be the small size of the screen and/or the use of a digitizer pen as the input device. If the physician reoriented the tablet towards the patient, he would likely be unable to see the screen himself and would not be able to use the digitizer pen to input entries like keywords to search for the needed information or to review the patient's medical record. Moreover, since the tablet is slightly tilted as shown in Figure 2, it would be more difficult for the patient to peep at the screen when the tablet was oriented towards the physician. Hence, the tablet computer did not support collaborative viewing of the screen in the same way as the desktop computer. On the other hand, the nature of the scenario might also have motivated the physician's technology withdrawal. For example, in scenario 3, physician 2 was using the tablet to search for information on genetic causes of esophageal cancer and, due to the sensitive nature of the topic, the physician may not have wanted the patient to see it before he had a chance to digest and possibly filter that information, which is consistent with previous research that care must be exercised when sharing sensitive health information [28].

Whether the physicians re-oriented the tablet towards the patients or themselves, the physicians in the study appreciated the ability to reorient the tablet, as physician 1 commented:

\section{"The advantage of this is it does allow you to kind of position it in a way that you can do it privately so I quite like that."}

The positioning of the tablet and the use of digitizer pen also allowed the physicians to interact with both the patient and the device without changing their bodily orientation. Consequently, they could easily re-establish eye contact with the patient when they looked up from the screen, an advantage over the desktop computer.

Thus, the mobile device affords flexible control and re-orientation for the physicians to share information with the patients while its movability also made it convenient for the physicians to
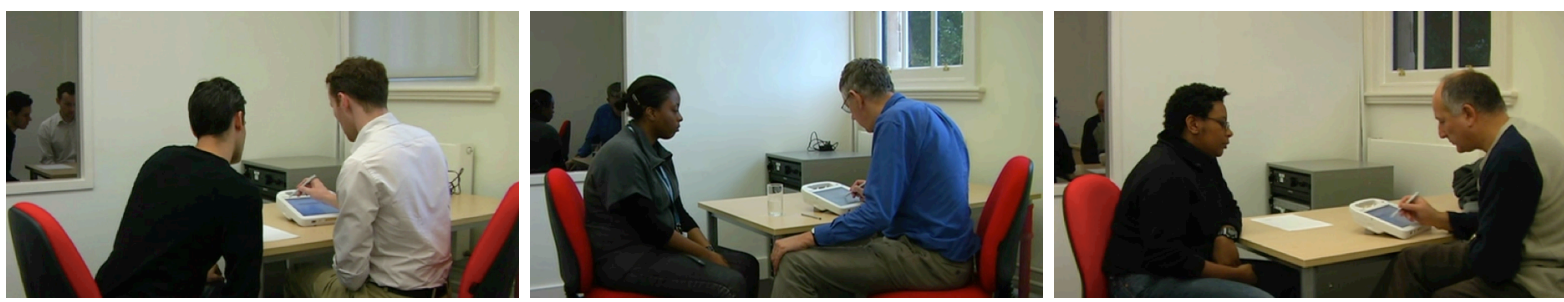

Figure 2: (left) Physician turned tablet slightly towards patient (active information sharing), (center \& right) Physicians turned tablet towards themselves (technology withdrawal). 

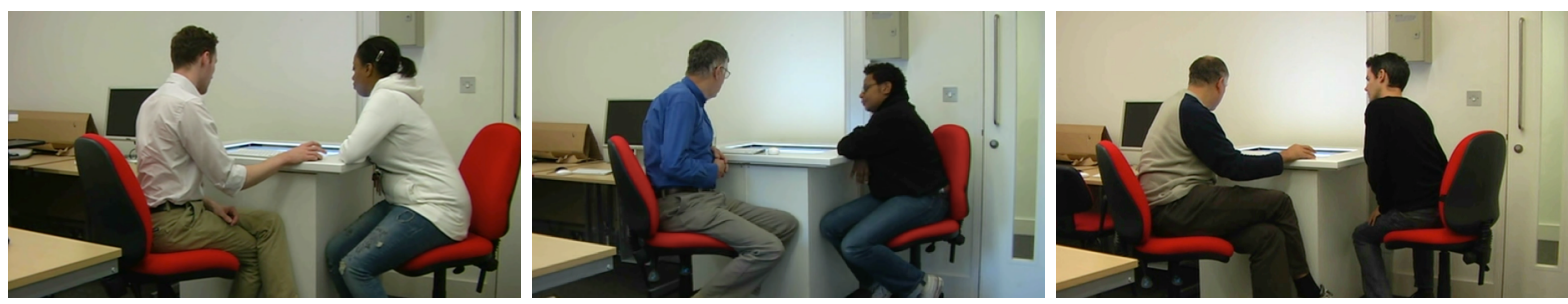

Figure 3: Physician and patient both look at the screen of the tabletop computer.

withdraw the technology from the patients when needed, such as when sensitive information was displayed.

\subsection{Tabletop computer}

Tabletop computers have received attention within the computer science field as a device that enables and promotes collaboration and its use has been explored in healthcare settings [27]. Since the screen is flat, the physician could easily re-establish eye contact with the patient without being interrupted by the screen. As with the tablet, this could be considered to be a significant advantage over the desktop computer. Another advantage of the tabletop is its large screen, which made it easier for both physicians and patients to read from the screen. Physicians 2 and 3 both demonstrated passive information sharing during the consultations through subtle mouse movement, presumably because they felt that they did not need to encourage the patients to look at the screen since it was readily visible to the patient. It was also interesting to note that physician 3's gaze could effectively direct patient's gaze movements towards specific information on the screen, and all the patients took advantage of the ledge around the tabletop to help them lean forward to gain a better view of the screen.

While all three patients looked down at the screen during the consultations, one had to change his bodily orientation so that he was able to see the screen from the same angle as the physician (Figure 3, right), suggesting that not being able to re-orient the screen or the content may be a limitation of the particular tabletop computer that was used. In addition, all physicians commented that the flat screen was not easy to view from the sitting position, suggesting a slightly tilted screen, like the tablet, may be needed to facilitate information access.

With a large flat screen like a traditional table, we had anticipated that physicians would make extensive use of pointing or gesturing to guide the patients' gaze to the screen but only physician 1 pointed at the information on the screen to share with the patient whereas the other physicians only used gaze or mouse movement to direct the patients' gaze.

Having an open shared space between patient and provider also raised some concerns regarding privacy. As physician 2 mentioned:

"You have to remember one issue about who sees the screen, sometimes people come in with a relative... the positioning of the relative, the patient, the doctor and the screen is rather critical and what you need is a situation where the relative doesn't see what's on the screen but the patient can."

To control information sharing, physicians in the study appeared to make spatial use of the tabletop screen, which is similar to the designation of "personal, group, and storage" territories identified in [34]. For example, physician 2 had the patient record displayed on the left side of the tabletop far away from the patient, whereas the browser for searching information was displayed close to the patient. Thus, although the tabletop screen offered an open, unobstructed view to both the physicians and the patients, physicians were able to make use of territoriality on the tabletop surface to manage information sharing to some extent.

In summary, our study of the three different devices in the exam room reveals that physicians can somewhat control information sharing with patients by adjusting the orientation of the desktop computer screen or the spatial location of information displayed on the tabletop computer. In both situations, patients may also lean forward or adjust their bodily orientation to view the information on the screen. On the other hand, the mobile tablet computer affords flexibility and easy orientation for physicians to share or withdraw information with or from patients at discretion.

\section{Discussion}

This exploratory experimental study demonstrates that different computing devices shape the ways in 
which provider and patient interact during the consultation. In particular, the choice of device influences the physician's gaze, bodily orientation, and screen sharing with the patient. As described in the findings section, no system is perfect; instead, physicians preferred certain features of each device. In this section, we first summarize the preferred features of the computing devices and how they can better support patient-centered communication. Next, we discuss how the concept of territoriality can be applied to the design of a tabletop computer to facilitate patient-physician communication, balancing the control of openness and privacy. We then present the limitations of the study.

\subsection{Device features for supporting patient- centered communication}

Desktop computers afford physicians to share information with patients by re-orienting the screen towards the patient, and also by explicit pointing at the screen to direct patients' gaze (active information sharing). Physicians were also observed to share information when patients were able to follow the physicians' gaze on the screen. On the other hand, patients lean forward and/or re-orient themselves in order to view the screen when physicians do not reorient the screen towards the patients. Desktop computers may also be oriented in such an angle that the screen is not visible to the patients (technology withdrawal) but we did not observe this in our study.

Large screens are preferred since it is easier to share information with the patient although extra effort may be needed to guide the patient's gaze by pointing and signaling to the screen. Patients were also found to be able to follow the subtle gaze of the physicians during information sharing. However, the easy sharing capability afforded by a large screen raises a privacy concern, since relatives and friends can also view the screen easily. Therefore, physicians also valued the ability to re-orient the screen to different angles so that they can have more control to either share or hide the screen based on the information displayed and on the personnel present during the consultation. Lastly, although an upright screen is not desired since it can block eye contact, a completely horizontal screen was found difficult for providers to see information on the screen, suggesting a slightly tilted or adjustable screen is needed.

Moreover, the findings of the study suggest that, instead of having one display mode throughout an entire consultation, providers desire both openness and privacy when engaging with computing devices, and flexibility in the device that allows them to control what information is revealed or hidden. This is reflected in the active information sharing and technology withdrawal behaviors observed in the study, as providers exert control over the ways in which information is shared during consultations based on the specific situation. The findings suggest that future computing devices designed for exam rooms should consider these characteristics and provide a large screen that allows flexible manipulation during the consultation.

\subsection{Territoriality and information sharing}

Patient-centered care aims to encourage patients to become active collaborators in their own healthcare. Thus effective patient-physician communication and information sharing is crucial in the exam room. Our research indicates that a large adjustable horizontal screen has the potential to afford openness in information sharing, and the ability to preserve privacy and filtering of sensitive information. We thus propose to implement the concept of territoriality in collaborative workspaces [34] in the design of information devices for the exam room. This design also corresponds to the physicians' intuitive tabletop usage of spatial areas identified in our study.

Territoriality is a means "to assert some level of control or ownership over a space" and "to maintain a desired level of personal space and privacy" [35]. We suggest providing designated information territories on the tabletop computer to better support information sharing and privacy protection.

As in our study, a tabletop computer can be used as a single shared workspace. However it does not address the issue of privacy in patient-physician communication. Rather, a tabletop partitioned into territories in which information is only visible to designated personnel based on their spatial locations can be used to control information access so that it can address both issues of openness and privacy. To enhance patient-centered communication, we can design the tabletop to allow dynamic partitioning into physician, patient, and friend territories.

Information, such as medical records and lab results, is first retrieved and displayed in the physician territory that is only visible to the physician. The physician then reviews and filters the information before sending a copy of the information suitable for the patient to appear in the patient territory, which can be re-oriented for the patient to view. The physician can also see the information in the personal territory so that patient-physician dialog may continue over the information. When other personnel, such as relatives or friends of the patient, 
are present, the patient can send a copy of selected information from the patient territory to appear in the friend territory for them to see. Alternatively, a mobile tablet computer may be leveraged to serve as the friend territory, in the same way that information was copied from Augmented Surfaces [29] and UbiTable [37] to an adjacent laptop computer. This design making use of territoriality allows seamless sharing of information that can facilitate patientcentered communication in the exam room, with flexible control over openness and privacy.

\subsection{Limitations}

The findings of this small exploratory pilot study may not be generalizable, especially given that existing studies of communication around health IT emphasize how computer use and subsequent communication varies according to provider [1][29]. However, the use of a within-participant design did allow us to distinguish between those behaviors that are associated with a particular physician, in contrast to those that appear to be more associated with a particular device. Generalizability is also limited by the nature of the scenarios. As described above, the patients were actors and the physicians were aware of this and the fabricated scenarios which were designed to specifically encourage searching and sharing of information by the physician, and are not intended to be representative of the range of cases a primary care physician may encounter in their work.

\section{Conclusion}

To the best of our knowledge, this is the first study to consider the impact of choice of computing device on patient-provider communication in the exam room. The findings reveal that the choice of device impacts the extent to which patient-provider interaction during the consultation is patientcentered. To better support patient-centered communication, a large horizontal screen offers an open, unobstructed view to both the physician and the patient so that they can more easily re-establish eye contact, which can encourage patient engagement and active participation. More generally, the findings suggest the need for design of future systems to incorporate the characteristics of both openness and privacy to provide both flexibility and control. To do this, we propose partitioning the screen into physician, patient, and friend territories for seamless information sharing. In addition, the findings indicate that this is a topic worthy of further exploration, both through experimental studies and longer term in situ evaluations.

\section{Acknowledgements}

We would like to thank the physicians who participated in the study and Dr. Peter Davis who assisted us in the development of the scenarios.

\section{References}

[1] Alsos, O.A., Das, A. and Svanæs, D. Mobile health IT: The effect of user interface and form factor on doctor-patient communication. International Journal of Medical Informatics. 2012, 81(1):12-28.

[2] Asan, O. and Montague, E. Technology-mediated information sharing between patients and clinicians in primary care encounters. Behaviour \& Information Technology. 2014B, 33(3):259-70.

[3] Asan, O.D., Smith, P. and Montague, E. More screen time, less face time - implications for EHR design. Journal of Evaluation in Clinical Practice. 2014A, 20(6):896-901.

[4] Asan, O., Young, H.N., Chewning, B. and Montague, E. How physician electronic health record screen sharing affects patient and doctor non-verbal communication in primary care. Patient Education and Counseling. 2015 3;98(3):310-6.

[5] Ash J, Berg M \& Coiera E. Some unintended consequences of information technology in health care: the nature of patient care information systemrelated errors. JAMIA 2004, 11(2):104-12.

[6] Bates, D.W., Teich, J.M., Lee, J., et al. The impact of computerized physician order entry on medication error prevention. J Am Med Inform Assoc. Jul-Aug, 6(4):313-21.

[7] Chaudhry B, Wang J, Wu S, et al. Systematic review: impact of health information technology on quality, efficiency, and costs of medical care. Ann Intern Med 2006,144:742-52.

[8] Chen, Y. Documenting transitional information in EMR. Proceedings of CHI 2010, 1787-1796.

[9] Coiera, E. (2000). When conversation is better than computation. Journal of American Medical Informatics Association, 7:277-286.

[10] Crosson J, Ohman-Strickland P, Hahn K, DiCiccoBloom B, Shaw E, Orzano A, John C \& Benjamin F. Electronic medical records and diabetes quality of care: results from a sample of family medicine practices, Annals of Family Medicine 2007, 5:209215 .

[11] Epstein RM, Street RL. The Values and Value of Patient-Centered Care. The Annals of Family Medicine. 2011 March 1, 2011;9(2):100-3.

[12] Frankel R, Altschuler A, George S, et al. Effects of Exam-Room Computing on Clinician-Patient 
Communication: A Longitudinal Qualitative Study. Journal of General Internal Medicine. 2005;20(8):67782.

[13] Gibson M, Jenkings KN, Wilson R, Purves I. Multitasking in practice: Coordinated activities in the computer supported doctor-patient consultation. International Journal of Medical Informatics. 2005 2005;74:425-36.

[14] Greenfield S., Kaplan S. and Ware, J.E. Jr. (1985). Expanding patient involvement in care. Effects on patient outcomes. Ann Intern Med. Apr;102(4):520 28

[15] Heath, C. and Nicholls, K. Body Movement and Speech in Medical Interaction, Cambridge University Press, Cambridge, 1986.

[16] Hsu, J., Huang, J., Fung, V., Robertson, N., Jimison, H., \& Frankel, R. (2005). Health information technology and physician-patient interactions: impact of computers on communication during outpatient primary care visits. Journal of the American Medical Informatics Association, 12(4), 474-480.

[17] Institute of Medicine Committee on Quality of Health Care in America. Crossing the quality chasm: A new health system for the 21st century: National Academy Press; 2001.

[18] Knapp, M.L., Hall, J.A. and Horgan, T.G. Nonverbal Communication in Human Interaction. $8^{\text {th }}$ Ed., Cengage Learning US, 2013.

[19] Kumarapeli P, de Lusignan S. Using the computer in the clinical consultation; setting the stage, reviewing, recording, and taking actions: multi-channel video study. Journal of the American Medical Informatics Association. 2013 June 1, 2013;20(e1):e67-e75.

[20] Lelievre S, Schultz K. Does computer use in patientphysician encoun- ters in uence patient satisfaction? Can Fam Physician 2010;56(1):e6- e12.

[21] Li, X. (2014). Disease communication and trust build: A research on the interaction of doctor-patient on social media (Master). Ji'nan University, Ji'nan, People's Republic of China.

[22] Liang, Q., Li, X., Liu, B. and Liu, X. (2016). Exploration of communication's effect on doctorpatient relationship. Science \& Technology Vision, (4):12-13.

[23] Luff, P., Heath, C. and Greatbatch, D. (1992). Tasksin-interaction: paper and screen-based documentation in collaborative activity. Proceedings of the Conference on Computer Supported Cooperative Work, 163-170.

[24] McGrath JM, Arar NH, Pugh JA. The influence of electronic medical record usage on nonverbal communication in the medical interview. Health Informatics Journal. 2007 June 1, 2007;13(2):105-18.

[25] Miles M, Huberman A. Early steps in analysis. Qualitative data analysis: an expanded sourcebook 2nd ed. Thousand Oaks, California: SAGE Publications; 1994. p. 50-89.
[26] Montague E, Asan O. Dynamic modeling of patient and physician eye gaze to understand the effects of electronic health records on doctor-patient communication and attention. International Journal of Medical Informatics. 2014;83(3):225-34.

[27] Piper AM. Supporting medical communication with a multimodal surface computer. CHI '10 Extended Abstracts on Human Factors in Computing Systems. Atlanta, Georgia, USA: ACM; 2010. p. 2899-902.

[28] Rajabiyazdi, F., Perin, C., Vermeulen, J., MacLeod, H., Gromala, D., \& Carpendale, S. (2017). Differences That Matter: In-Clinic Communication Challenges. Pervasive Health.

[29] Rekimoto, J. \& Saitoh, M. (1999). Augmented Surfaces: A Spatially Continuous Work Space for Hybrid Computing Environments. Proc. of CHI '99, pp. 378-385.

[30] Rhodes P, Small N, Rowley E, Langdon M, Ariss S, Wright J. Electronic Medical Records in Diabetes Consultations: Participants' Gaze as an Interactional Resource. Qualitative Health Research. 2008 September 1, 2008;18(9):1247-63.

[31] Ritchie J, Spencer L. Qualitative data analysis for applied policy research. In: Bryman A, Burgess RG, editors. Analyzing qualitative data. London: Routledge; 1994.

[32] Rudkin, S.E., Langdorf, M.I., Macias, D., Oman, J.A. and Kazzi, A.A. (2006). Personal digital assistants change management more often than paper texts and foster patient con dence. European Journal of Emergency Medicine, 13(2):92-6.

[33] Saleem JJ, Flanagan ME, Russ AL, et al. You and me and the computer makes three: variations in exam room use of the electronic health record. Journal of the American Medical Informatics Association. 2014 February 1, 2014;21(e1):e147-e51.

[34] Scott, S. Territoralitity in Collaborative Tabletop Workspaces. $\mathrm{PhD}$ thesis, University of Calgary, Calgary, Alberta, Canada, March, 2005.

[35] Stacey D. Scott and Sheelagh Carpendale. Theory of Tabletop Territoriality. In C. Müller-Tomfelde (Ed.) Tabletops - Horizontal Interactive Displays, Springer, 2010, 375-406.

[36] Shachak A, Reis S. The impact of electronic medical records on patient-doctor communication during consultation: a narrative literature review. Journal of Evaluation in Clinical Practice. 2009;15(4):641-9.

[37] Shen, C., Everitt, K.M., \& Ryall, K. (2003). UbiTable: Impromptu Face-to-Face Collaboration on Horizontal Interactive Surfaces. Proc. of UbiComp'03, pp. 281288.

[38] Stewart M.A. (1995). Effective physician-patient communication and health outcomes: a review. CMAJ. 152:1423-33.

[39] Strayer, S., Semier, M., Kington, M. and Tanabe, K.O. (2010). Patient Attitudes Toward Physician Use of 
Tablet Computers in the Exam Room. Journal of Family Medicine, 42(9):643-647.

[40] Sun, N. and Rau, P.L. (2017). Barriers to Improve Physician-Patient Communication in a Primary Care Setting: perspectives of Chinese physicians. Health Psychology and Behavioral Medicine, 5(1):166-176.

[41] Tang, C. and Carpendale, S. Evaluating the deployment of a mobile technology in a hospital ward. CSCW 2008, 205-214.

[42] White A, Danis M. Enhancing patient-centered communication and collaboration by using the electronic health record in the examination room. JAMA. 2013, 309(22):2327-8. 